\title{
Design of Tunable Multiband Hybrid Graphene Metal Antenna in Microwave Regime
}

\author{
Hussein A. Abdulnabi, Yasin Yousif Al-Aboosi \\ Department of Electrical Engineering, AL- Mustansiryah University, Baghdadi, Iraq
}

\begin{tabular}{l}
\hline \hline Article Info \\
\hline Article history: \\
Received Mar 17, 2018 \\
Revised Jul 27, 2018 \\
Accepted Aug 21, 2018 \\
\hline
\end{tabular}

\section{Keywords:}

Graphene

Microwave antenna

Tunable antenna

Multi-band

Reconfigurable antenna

\begin{abstract}
Graphene is an allotrope (form) of carbon consisting of a single layer of carbon atoms arranged in an hexagonal lattice. It is the basic structural element of many other allotropes of carbon, such as graphite, charcoal, carbon nanotubes and fullerenes. In this paper, a tunable hybrid metalgraphene antenna in the microwave regime is proposed. This antenna composed of the copper patch and four graphene strips. The antenna designs used for the cellular long-term evolution system and the operating frequency bands of $1.8,2.5,2.6$, and $3.6 \mathrm{GHz}$, are evaluated to demonstrate the working principle and the performance tradeoffs. Furthermore, the proposed antenna can be tuned by varying applied DC voltage on the graphene which leads to change in the chemical potential of the graphene and hence the surface conductivity and electrical properties are changed. The simulation results reveal that the antenna operates in multi-band where scattering factor $\mathrm{S} 11<$ $10 \mathrm{~dB}$. In addition, the results show that hybrid metal-graphene frequency reconfigurable antennas can, at the same time, provide a tunable bandwidth and antenna matching.
\end{abstract}

Copyright $(0) 2018$ Institute of Advanced Engineering and Science. All rights reserved.

\section{Corresponding Author:}

Hussein A,

Department of Electrical Engineering,

AL- Mustansiryah University,

Baghdadi, Iraq.

Email: hussein_ali682@yahoo.com

\section{INTRODUCTION}

Graphene is a flat monoatomic layer of carbon atoms distributed in a 2-D honeycomb-likelattice [1]. Furthermore, an unbiased pristine single layer of graphene only absorbs $2.3 \%$ of visible light [2] and supports breaking forces of up to $42 \mathrm{~N} / \mathrm{m}$ with Young's modulus of $1 \mathrm{TPa}$ and an intrinsic strength of $130 \mathrm{GPa}$ [3] while also being extremely light $(0.77 \mathrm{mg} / \mathrm{m} 2)$. As a result, a reconfigurable antenna made of graphene could also be transparent, flexible, and lightweight. Graphene has been named the simplest complex material and has drawn attention due to its unique properties and advantages and it is used in many applications including electrical, thermal, and mechanical applications [4]. The surface conductivity of the graphene can be varied by varying applied electrical voltage [5], thus many reconfigurable graphene-based devices such as antennas, filters, absorbers and polarizer's have been proposed for the band in the microwave, $\mathrm{THz}$ and optical frequencies.

Future wireless devices will be accumulating multiple wireless services in one system operating over a wide frequency spectrum, such as third $(3 \mathrm{G})$ and fourth $(4 \mathrm{G})$ mobile generations and beyond at 700 $800 \mathrm{MHz}, 1.8-2.6 \mathrm{GHz}$, and $3.6 \mathrm{GHz}$; WIFI at 2.4, 3.6, and $5 \mathrm{GHz}$. To collect a multi-radio transmitting system in one system, a reconfigurable antenna is required to provides an option to integrate multiple radiating elements at different frequencies into a single physical antenna, and hence, save space. A reconfigurable antenna is designed to change its resonant frequency, operational bandwidth, radiation pattern, 
and/or polarization manually or automatically (via software) to respond to different radio system requirements, and the environment.

Graphene material can be used in a reconfigurable antenna due to its to changing the surface conductivity by applying external electric field. Importantly, the admittance of graphene can be tuned by applying an electrostatic field perpendicular to the graphene layer, or in other words, by applying a DC current voltage bias, and hence, high and low impedances can be set to get the ON and OFF states as in the switches.

This effect is used in this paper to varying the electrical dimension of the antenna, and consequently, change the resonant frequency. However, graphene is not only an alternative option to a conventional RF switch, it can be used for fabricating the radiating antenna itself. Graphene reconfigurable antennas are being mostly studied for infra-red and terahertz frequencies as graphene can significantly reduce the size and provide high antenna reconfigurability. At such frequencies [6-7]. Huang et al [8] studied the performance of wearable antennas made of graphene ink from 1 to $5 \mathrm{GHz}$. The resonant frequency, bandwidth, and gain of the antennas in their study were slightly affected by bending and twisting.

The proposed antennas in this paper might produce enough reconfigurability to compensate for these effects observed in the graphene ink case. Since antennas fully made of graphene are expected to have low antenna efficiencies with very little reconfigurability at microwave frequencies [9], the antenna designs proposed here are hybrid metal-graphene frequency reconfigurable antennas.

\section{BACKGROUND}

The graphene can display as an infinitesimally flimsy surface which is portrayed by surface conductivity $\sigma\left(\omega, \mu_{c} \gamma, T\right)$. The graphene conductivity can be written by Drude model [10]. The Kubo formula of the graphene conductivity can be written

$$
\begin{aligned}
& \sigma=\sigma_{\text {intra }}+\sigma_{\text {inter }} \\
& \sigma_{\text {intra }}\left(\omega, \mu_{\mathrm{c}}, \gamma, \mathrm{T}\right)=\frac{-\mathrm{ie}^{2} \mathrm{k}_{\mathrm{B}} \mathrm{T}}{\pi \hbar^{2}}\left[\frac{\mu \mathrm{c}}{\mathrm{k}_{\mathrm{B}} \mathrm{T}}+2 \ln \left(\mathrm{e}^{\frac{-\mu_{\mathrm{c}}}{\mathrm{k}_{\mathrm{BT}}}}+1\right)\right] \frac{1}{\omega-\mathrm{j} 2 \gamma} \\
& \sigma_{\text {inter }}\left(\omega, \mu_{\mathrm{c}}, \gamma, \mathrm{T}\right)=\frac{-\mathrm{je}}{4 \pi \hbar} \ln \left(\frac{2\left|\mu_{\mathrm{c}}\right|-(\omega-\mathrm{j} 2 \gamma) \hbar}{2\left|\mu_{\mathrm{c}}\right|+(\omega-\mathrm{j} 2 \gamma) \hbar}\right.
\end{aligned}
$$

Where, voltage

$\omega=$ Angular frequency in $\mathrm{rad} / \mathrm{second}$ and

$\gamma=$ Scattering rate in $\mathrm{s}-1$

$\mu_{c}=$ Chemical potential in $\mathrm{eV}$, which can be controlled by chemical doping or by applying a bias

$\mathrm{T}=$ Temperature in Kelvin

$\mathrm{e}=$ Electron charge

$\hbar=$ Reduced Planck's constant, and

$k_{B}=$ Boltzmann constant

The intra band term dominates the graphene conductivity over an ultra-wide band of frequencies. The real part of the intra band conductivity being much larger than the imaginary part in this frequency range.

Interband conductivity dominates in the high frequencies, its real part being much smaller than its imaginary part. Although the interband conductivity is much larger than the intraband conductivity in the $\mathrm{THz}$ range, both terms were taken into account for the calculation of the surface impedance. Graphene surface impedance can be obtained from its conductivity by using the equations.

$$
\mathrm{Z}_{\mathrm{S}=1 / \sigma(\omega)}=\mathrm{R}_{\mathrm{S}}\left(\mathrm{V}_{\mathrm{b}}\right)+\mathrm{j} \mathrm{X}_{\mathrm{S}}\left(\mathrm{V}_{\mathrm{b}}\right)
$$

\section{THE PROPOSED ANTENNA MODEL}

The proposed antenna consists of the patch from copper with four slots filled with graphene and transmission line at the middle of the bottom side of the patch. The antenna patch placed over $1 \mathrm{~mm}$ alumina and the graphene strips connected to DC voltage source with gate doped semiconductor (Sio2) connected to 
graphene strips via DC voltage source for the purpose of tunability of the antenna. The antenna substrate is a silicone material of $4 \mathrm{~mm}$ thickness and the antenna grounded by $0.035 \mathrm{~mm}$ copper layer. The antenna shape in CST program and layers of the antenna shown in Figure 1. A transmission line of a width of $2 \mathrm{~mm}$ is used for antenna feeding. Figure 2 shows the top view of the antenna and parameters symbols, Table 1 list the parameters dimensions in millimetres. Figure 2 shows the parameter symbol and Table 1 shows the parameter value in millimetre.

\begin{tabular}{c}
\hline Antenna patch with graphene \\
\hline Alumina Al2o3 \\
gate doped semiconductor Sio2 \\
Antenna Substrate $\mathrm{Si}$ \\
Antenna Ground copper \\
\hline
\end{tabular}

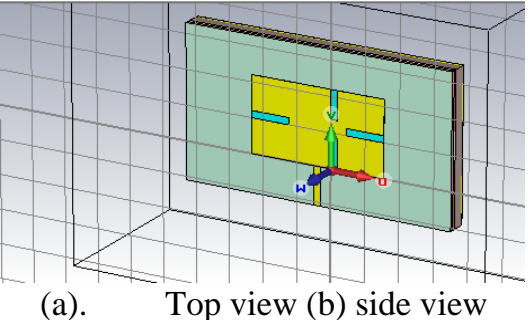

Figure 1. The proposed antenna

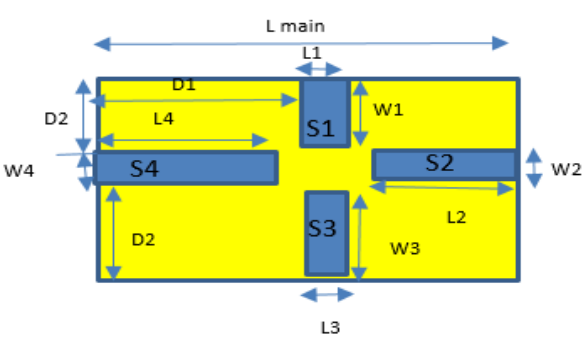

Figure 2.Top view of the proposed antenna

Table 1. antenna parameters dimensions

\begin{tabular}{cccc}
\hline Parameter symbol & Value $(\mathrm{mm})$ & Parameter symbol & Value $(\mathrm{mm})$ \\
\hline L1 & 2 & W1 & 8.5 \\
L2 & 11 & W2 & 2 \\
L3 & 2 & W3 & 8.5 \\
L4 & 11 & W4 & 2 \\
L main & 39 & & \\
D1 & 23.5 & & \\
D2 & 10 & & \\
\hline
\end{tabular}

\section{RESULTS OF THE PROPOSED ANTENNA}

All antenna configurations are simulated using a Computer Simulation Technology (CST) Microwave studio (MWs) software package which is based on Finite Integration Technique (FIT) instead of MoM. Many cases simulated and tested (some or all graphene strips connected to certain voltage and other strips connected to zero volts) and the next section shows the result. When the DC voltage connected to graphene strip, the graphene strip in ON state. When the applied DC voltage equal zero, the graphene strip in OFF state.

\subsection{Case 1: S1, S3 On, S2, S4 Off}

Figure 3 shows the far field of the proposed antenna at frequencies ( $\mathrm{f}=1.8,2.4,2.5,2.6,3.6,5 \mathrm{GHz})$ when S1, S3 on and S2, S4 off. Figure 4 shows the scattering parameter of the antenna. The antenna has three resonance frequencies 3.5, 4.2, $4.4 \mathrm{GHz}$. Figure 5 shows the gain of the antenna. The antenna has a good gain at frequency range $(2-6) \mathrm{GHz}$.

\subsection{Case 2: S1, S2, S4 OFF, S3 ON}

Figure 6 shows the far field of the proposed antenna at frequencies $(\mathrm{f}=3.6,4.2,4.4,5.6 \mathrm{GHz})$ when S3 ON and S1 S2,S4 OFF. Figure 7 shows the scattering parameter of the antenna. The antenna has three resonance frequencies 3.5, 4.2, $4.4 \mathrm{GHz}$. Figure 8 shows the gain of the antenna. The antenna has a good gain at frequency range $(2-6) \mathrm{GHz}$.

\subsection{Case 3: S1, S2, S3, S4 OFF,}

Figure 9 shows the far field of the proposed antenna at frequencies $(\mathrm{f}=3.6,4.2,4.4,5.6 \mathrm{GHz})$ when and S1 S2,S3,S4 OFF. Figure 10 shows the scattering parameter of the antenna. The antenna has three 
resonance frequencies 3.5, 4.2, $4.4 \mathrm{GHz}$. Figure 11 shows the gain of the antenna. The antenna has a good gain at frequency range (2.5-6) $\mathrm{GHz}$.

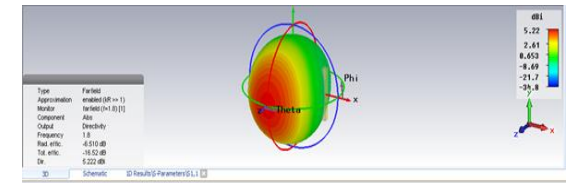

Far-field at $\mathrm{f}=1.8 \mathrm{GHz}$

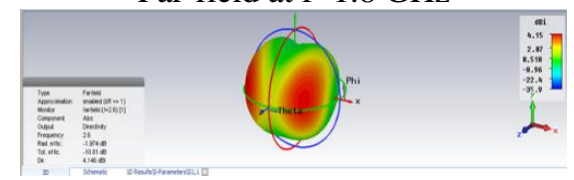

Far-field at $\mathrm{f}=2.6 \mathrm{GHz}$

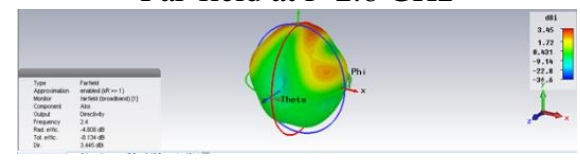

Far-field broadband $\mathrm{f}=2.4$

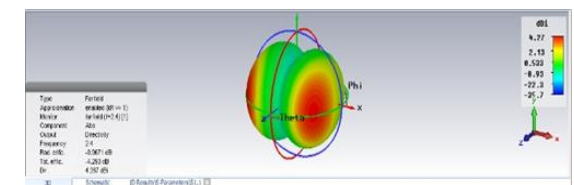

Far-field at $\mathrm{f}=2.4 \mathrm{GHz}$

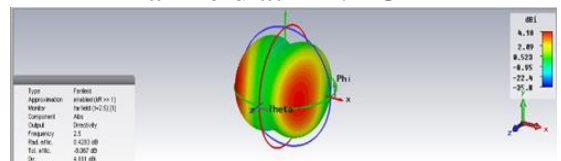

Far-field at $\mathrm{f}=2.5 \mathrm{GHz}$

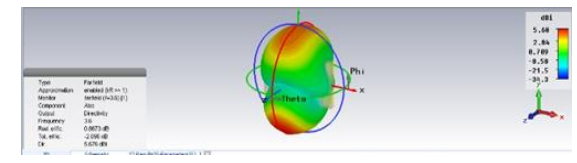

Far-field at $\mathrm{F}=3.6 \mathrm{GHz}$

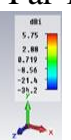

Far-field at $\mathrm{f}=5 \mathrm{GHz}$

Figure 3. Far field of the proposed antenna at frequencies ( $\mathrm{f}=1.8,2.4,2.5,2.6,3.6,5 \mathrm{GHz}$ )

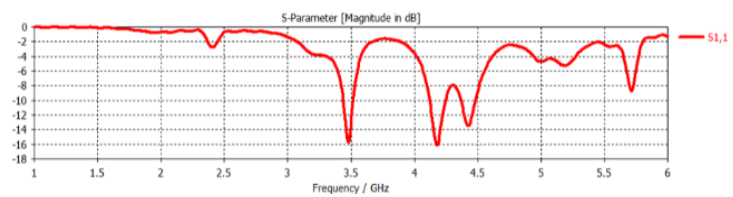

Figure 4. Scattering parameter S11 of the proposed antenna

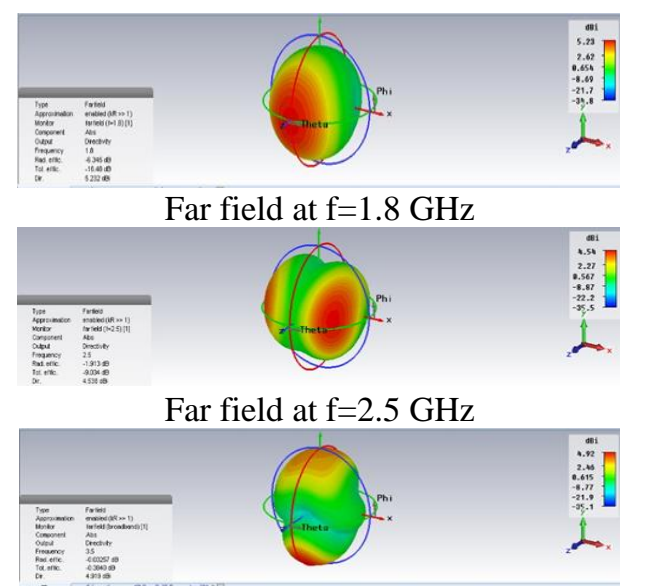

Far field at $\mathrm{f}=3.5 \mathrm{GHz}$

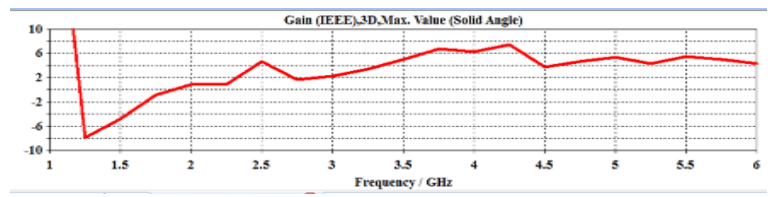

Figure 5. Gain of the proposed antenna
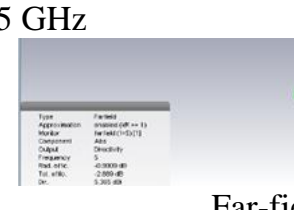

Far-field at $\mathrm{f}=5 \mathrm{GHz}$

Figure 6. Far field of the proposed antenna at frequencies $(\mathrm{f}=1.8,2.4,2.5,2.6,3.6,5 \mathrm{GHz})$ 


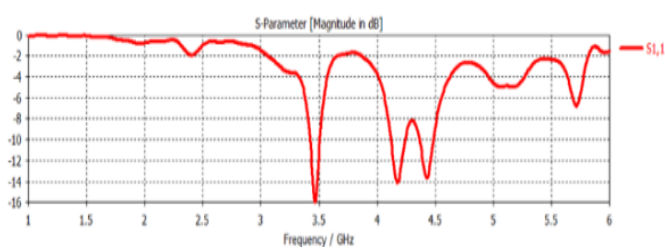

Figure 7. scattering parameter S11 of the proposed antenna

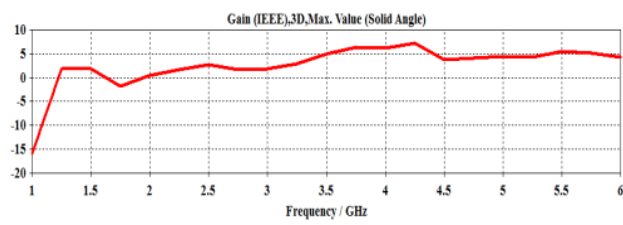

Figure 8. gain of the proposed antenna

\subsection{Case 4: S1, S2, S3, S4 ON,}

Figure 12 shows the far field of the proposed antenna at frequencies ( $\mathrm{f}=3.6,4.2,4.4,5.6 \mathrm{GHz})$ when and S1 S2, S3, S4 ON. Figure 13 shows the scattering parameter of the antenna. The antenna has three resonance frequencies 3.5, 4.2, 4.4 GHz. Figure 14 shows the gain of the antenna. The antenna has a good gain at frequency range (1.5-6) GHz.

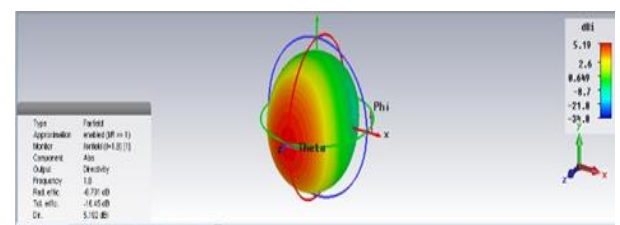

Far field at $\mathrm{f}=1.8 \mathrm{GHz}$

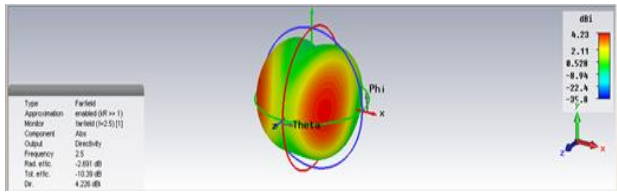

Far field at $\mathrm{f}=2.5 \mathrm{GHz}$

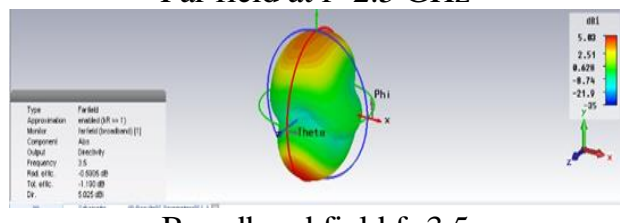

Broadband field $\mathrm{f}=3.5$

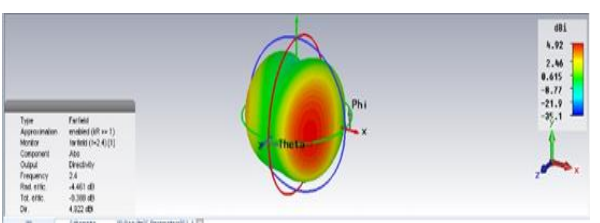

Far field at $\mathrm{f}=2.4 \mathrm{GHz}$

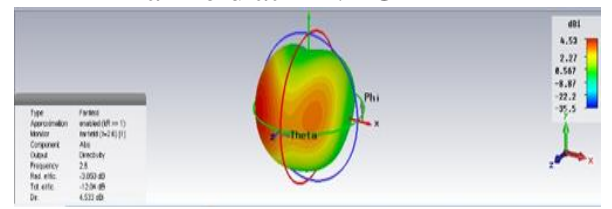

Far field at $\mathrm{f}=2.6 \mathrm{GHz}$

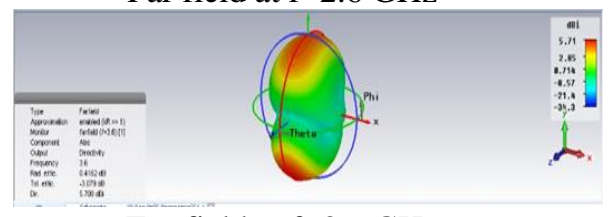

Far-field at $\mathrm{f}=3.6 \mathrm{GHz}$

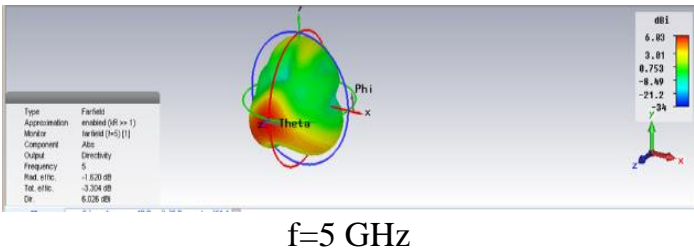

Figure 9. far field of the proposed antenna at frequencies $(\mathrm{f}=1.8,2.4,2.5,2.6,3.6,5 \mathrm{GHz})$

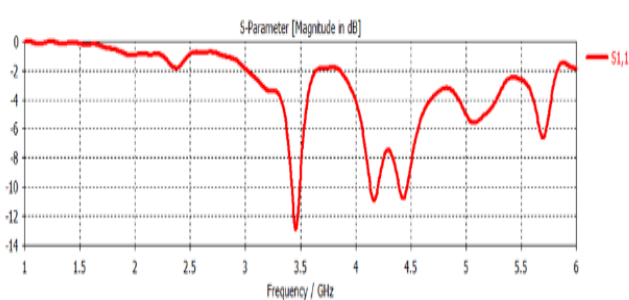

Figure 10. scattering parameter S11 of the proposed antenna

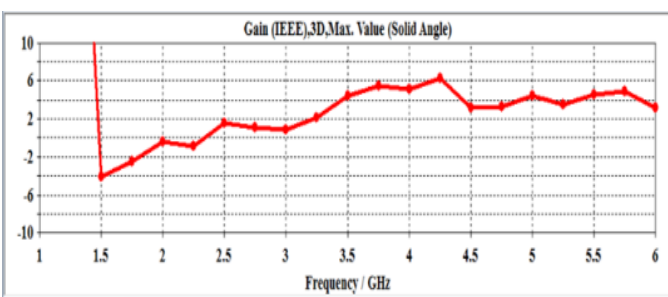

Figure 11. gain of the proposed antenna 


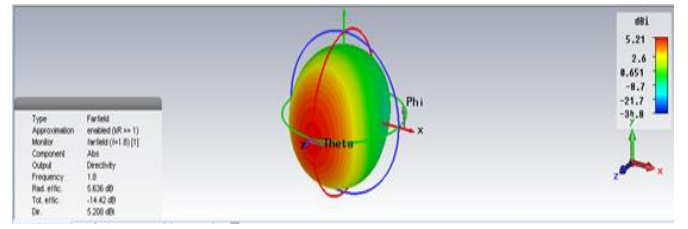

Far field at $\mathrm{f}=1.8 \mathrm{GHz}$

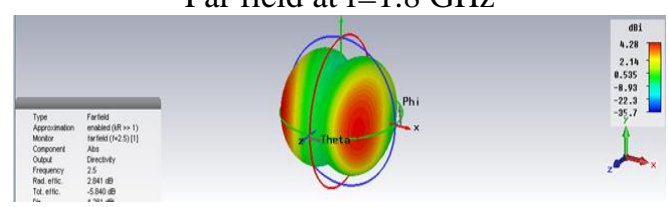

Far field at $\mathrm{f}=2.5 \mathrm{GHz}$

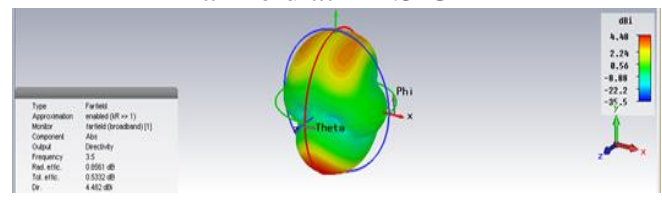

Far field at $\mathrm{f}=3.5 \mathrm{GHz}$

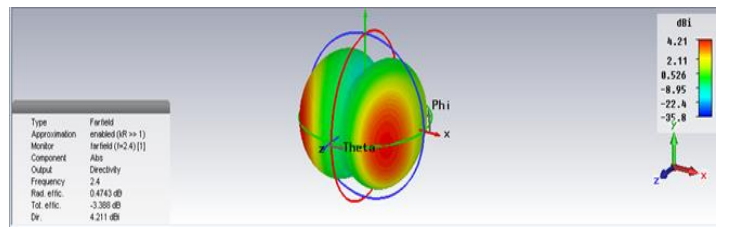

Far field at $\mathrm{f}=2.4 \mathrm{GHz}$

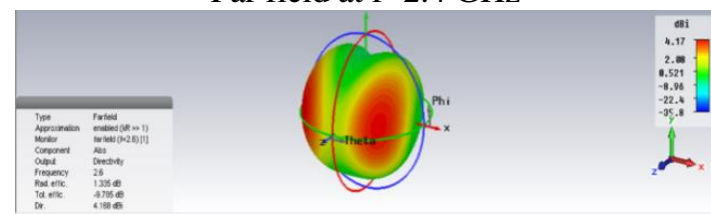

Far field at $\mathrm{f}=2.6 \mathrm{GHz}$

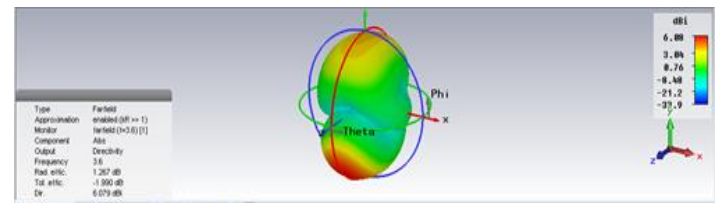

Far field at $\mathrm{f}=3.6 \mathrm{GHz}$

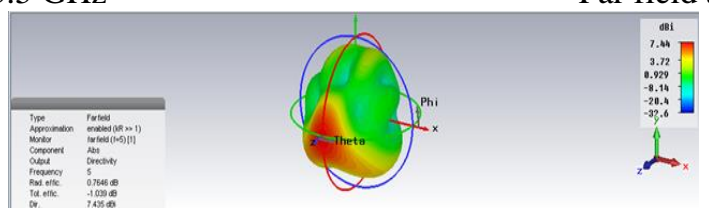

Far field at $\mathrm{f}=5 \mathrm{GHz}$

Figure 12. far field of the proposed antenna at frequencies $(\mathrm{f}=1.8,2.4,2.5,2.6,3.6,5 \mathrm{GHz})$

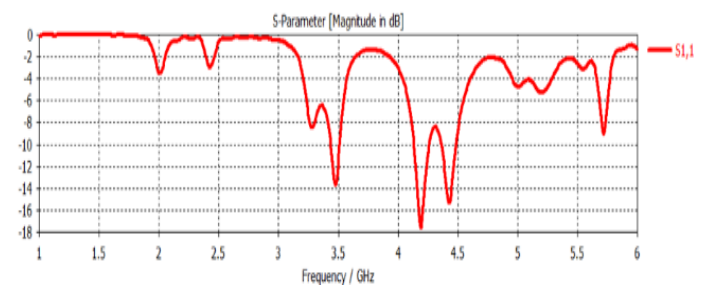

Figure 13. scattering parameter S11 of the proposed antenna

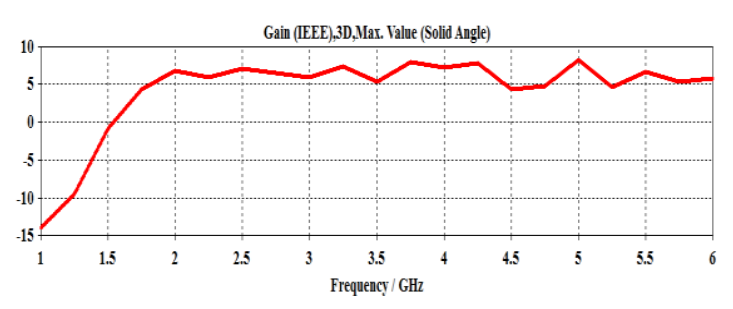

Figure 14. gain of the proposed antenna

\section{CONCLUSION}

In this paper, a reconfigurable hybrid metal-graphene antennas have been designed and simulated in microwave regime using CST Studio package. Many cases are taken, some or all graphene strips are ON and other strips or all are OFF. The designed antenna has a good gain and many resonance frequencies in themicrowave regime. The resonance frequencies and far field of the antenna variable in each case, thus we get a reconfigurable antenna.

\section{REFERENCES}

[1] C. N. Álvarez, R. C., J. S. Thompson, "Performance Analysis of Hybrid Metal-Graphene Frequency Reconfigurable Antennas in the Microwave Regime", IEEE transactions antennas and propagation, Vol. 65, No. 4, April 2017.

[2] F. Bonaccorso, Z. Sun, T. Hasan, and A. C. Ferrari, "Graphene photonics and optoelectronics," Nature Photon., vol. 4, no. 9, pp. 611-622, Sep. 2010.

[3] C. Lee, X. Wei, J. W. Kysar, and J. Hone, "Measurement of the elastic properties and intrinsic strength of monolayer graphene," Science, vol. 321, no. 5887, pp. 385-388, 2008.

[4] K. Geim. "Graphene: Status and prospects", Science, 324(5934), Jun. 2009, pp. 1530-1534,

[5] N. Grigorenko, M. Polini, and K. S. Novoselov. "Graphene plasmonics”, Nature Photon., 2012, 487, pp. 749-758. 
[6] Sensale - Rodr'iguez, R. Yan, L. Liu, D. Jena, and H. G. Xing. "Graphene for reconfigurable terahertz optoelectronics", Proc. IEEE, 101(7), Jul. 2013, pp. 1705-1716.

[7] H. A. Abdulnabi1, R. T. Hussein and R.S. Fyath, "0.1-10 thz single port log periodic antenna design based on hilbert graphene artificial magnetic conductor". ARPN Vol. 12, NO. 4, Feb. 2017

[8] M. Dragoman, A. A. Müller, D. Dragoman, F. Coccetti, and R. Plana, "Terahertz antenna based on graphene," J. Appl. Phys., vol. 107, no. 10, p. 104313, 2010.

[9] J. S. Gomez-Diaz and J. Perruisseau-Carrier, "Microwave to $\mathrm{THz}$ properties of graphene and potential antenna applications," in Proc. Int.Symp. Antennas Propag. (ISAP), Nov. 2012, pp. 239-242.

[10] X. Huang et al., "Highly flexible and conductive printed graphene for wireless wearable communications applications," Sci. Rep., vol. 5, p. 18298, Dec. 2015.

[11] J. S. Gomez-Diaz and J. Perruisseau-Carrier, "Microwave to THz properties of graphene and potential antenna applications," in Proc. Int.Symp. Antennas Propag. (ISAP), Nov. 2012, pp. 239-242.

[12] M. Dragoman, M. Aldrigo, A. Dinescu, D. Dragoman, and A. Costanzo "Towards a terahertz direct receiver based" 